\title{
Lesões de pele em idosos hospitalizados
}

\section{Skin injuries in hospitalized elderly}

\section{Lesiones de piel en ancianos hospitalizados}

Clóris Regina Blanski Grden¹, Taís Ivastcheschen", Luciane Patrícia Andreani Cabral',
Péricles Martim Reche1, Daniele Alaide de Siqueira de Oliveira², Danielle Bordin',

ORCID IDs

Grden CRB (D) https://orcid.org/0000-0001-6169-8826

Ivastcheschen T (iD https://orcid.org/0000-0001-8496-5990

Cabral LPA (D) https://orcid.org/0000-0001-9424-7431

Reche PM (iD https://orcid.org/0000-0001-7238-6318

Oliveira DAS (iD https://orcid.org/000-0002-4398-7963

Bordin D (iD) https://orcid.org/0000-0001-7861-0384

\section{COMO CITAR}

Grden CRB, Ivastcheschen T, Cabral LPA, Reche PM, Oliveira DAS, Bordin D. Lesões de pele em idosos hospitalizados. ESTIMA, Braz. J. Enterostomal Ther., 16:e4118. https://doi.org/10.30886/ estima.v16.639_PT

\section{RESUMO}

Objetivo: Identificar a prevalência de lesões de pele em idosos hospitalizados. Método: Pesquisa transversal com 202 idosos selecionados por conveniência e internados em um hospital do Paraná (Brasil). Os dados foram coletados entre setembro de 2017 e janeiro de 2018. Foram utilizados o Mini Exame do Estado Mental e o exame físico de enfermagem no idoso. Os dados foram submetidos à análise exploratória e descritos por frequência absoluta, percentual, médias e desvios-padrão. Resultados: predomínio de sexo feminino (51\%), faixa etária entre 60-69 anos (42,5\%), casados (52\%) e com baixa escolaridade (59,4\%). Identificou-se que 128 (63,4\%) idosos apresentavam couro cabeludo limpo; 116 (57,4\%), pele com coloração normal; 104 (51,5\%), turgor normal; 138 (68,3\%), temperatura normal; 160 (79,2\%), de espessura fina; 101 (50\%), textura macia; 144 (71,3\%), com cicatriz; 182 (90,1\%), sensibilidade; e 152 (75,3\%), prurido. Predominou índice de massa corporal eutrófico (38,6\%) e $176(87,1 \%)$ idosos apresentavam lesões de pele; 152 (75,3\%) possuíam lesões primárias e 151 (74,7\%), secundárias. Conclusão: o estudo identificou o alto de índice de lesões de pele em idosos internados. Espera-se que os resultados sensibilizem os enfermeiros quanto à importância do exame físico de pele como etapa fundamental para planejar ações de prevenção, monitoramento e cuidados.

DESCRITORES: Enfermagem geriátrica; Exame físico; Idoso; Hospitalização.

\footnotetext{
${ }^{1}$ Universidade Estadual de Ponta Grossa - Departamento de Enfermagem e Saúde Pública - Ponta Grossa/PR - Brasil.

${ }^{2}$ Mölnlycke Health Care Brasil - Almirante Tamandaré/PR - Brasil.

Autora correspondente: Clóris Regina Blanski Grden | Rua Teodoro Sampaio, 888 | CEP: 84.036-070 - Ponta Grossa/PR, Brasil |

E-mail: reginablanski@hotmail.com

Recebido: Set 012018 | Aceito: Dez 122018
} 


\begin{abstract}
Objective: To identify the prevalence of skin injuries in hospitalized elderly. Method: Cross-sectional study with 202 elderly patients selected for convenience and hospitalized in a hospital in Parana (Brazil). Data were collected between September 2017 and January 2018. The Mini-mental State Examination and the physical examination of nursing in the elderly were used. Data were submitted to the exploratory analysis and described by absolute frequency, percentage, means and standard deviations. Results: Women predominance (51\%), age range between 60-69 years (42.5\%), married (52\%) and low educational level (59.4\%). It was identified that 128 (63.4\%) elderly had clean scalp; 116(57.4\%), skin with normal coloring; 104 (51.5\%), normal turgor; 138 (68.3\%), normal temperature; 160 (79.2\%) of fine thickness; 101 (50\%), soft texture; 144 (71.3\%) with scar; 182 (90.1\%), sensitivity; and 152 (75.3\%), pruritus. Prevalence of eutrophic body mass index (38.6\%) and 176 (87.1\%) elderly had skin injuries; 152 (75.3\%) had primary injuries and 151 (74.7\%), secondary injuries. Conclusion: The study identified the high index of skin injuries in hospitalized elderly. The results are expected to make nurses aware of the importance of the physical examination of the skin as a fundamental step in planning preventive, monitoring and care actions.
\end{abstract}

DESCRIPTORS: Geriatric nursing; Physical exam; Elderly; Hospitalization.

\title{
RESUMEN
}

Objetivo: Identificar la prevalencia de lesiones de piel en ancianos hospitalizados. Método: Investigación transversal con 202 ancianos seleccionados por conveniencia e internados en un hospital de Paraná (Brasil). Los datos fueron recolectados entre septiembre del 2017 y enero del 2018. Fueron utilizados el Mini Examen del Estado Mental y el examen físico de enfermería en el anciano. Los datos fueron sometidos al análisis exploratorio y fueron descritos por frecuencia absoluta, porcentual, medias y desvíos-estándar. Resultados: predominio del sexo femenino (51\%), edad entre 60-69 años (42,5\%), casados (52\%) y con baja escolaridad (59,4\%). Se identificó que 128 (63,4\%) ancianos presentaron el cuero cabelludo limpio; 116 (57,4\%) piel con coloración normal; 104 (51,5\%), turgencia normal; 138 (68,3\%), temperatura normal; 160 (79,2\%) de espesura fina; 101 (50\%), textura suave; 144 (71,3\%), con cicatriz; 182 (90,1\%), sensibilidad; y 152 (75,3\%), prurito. Predominó un índice de masa corporal eutrófico (38,6\%) y 176 (87,1\%) ancianos presentaban lesiones de piel; 152 (75,3\%) poseían lesiones primarias y 151 (74,7\%), secundarias. Conclusión: el estudio identificó un alto de índice de lesiones de piel en ancianos internados. Se espera que los resultados sensibilicen a los enfermeros con respecto a la importancia del examen físico de la piel como una etapa fundamental para planificar las acciones de prevención, monitoreo y cuidados.

DESCRIPTORES: Enfermería geriátrica; Examen físico; Anciano; Hospitalización.

\section{INTRODUÇÃO}

As mudanças que constituem e influenciam o processo de envelhecimento são complexas e apesar de esse fenômeno ser uma realidade mundial crescente, os serviços de saúde apresentam inúmeros desafios para atender às necessidades de cuidado à saúde desse segmento populacional.

Modificações psicológicas, funcionais e sociais interferem no processo de envelhecer, favorecendo a perda gradual das reservas fisiológicas, o maior risco de contrair doenças e o declínio geral na capacidade intrínseca do indivíduo ${ }^{1}$. Dentre as inúmeras modificações fisiológicas que ocorrem, destacam-se as alterações do sistema tegumentar, tais como: redução da espessura dérmica, perda de fibras elásticas, diminuição do tecido adiposo subcutâneo e redução dos capilares na pele ${ }^{2}$, entre outras, as quais podem contribuir para que o idoso se torne mais suscetível às lesões de pele.
Nutrição inadequada, dispositivos invasivos, força mecânica, excesso de umidade, uso de fraldas e imobilidade física são fatores de risco que predispõe o aparecimento das lesões ${ }^{3,4}$, as quais se caracterizam como uma das principais complicações que podem acometer pacientes hospitalizados ${ }^{5,6}$, especialmente os idosos.

Em geral, esse segmento etário tende a apresentar várias patologias, condição que contribui para a hospitalização, com maior tempo de internação e consequente aumento dos custos com saúde ${ }^{7}$. Nesse contexto, destaca-se a importância de o enfermeiro realizar o exame físico de pele no idoso com o direcionamento para a detecção precoce de lesões, traumas e outras patologias, as quais podem acentuar limitações funcionais, com repercussão para a autonomia, independência e qualidade de vida.

Compreende-se que no idoso hospitalizado, o desenvolvimento de lesões tegumentares configura-se 
como indicador negativo da qualidade da assistência ${ }^{8}$, a qual tem como responsabilidade a manutenção da integridade cutânea.

\section{OBJETIVO}

Diante do exposto, o objetivo desse estudo foi identificar a prevalência de lesões de pele em idosos internados em uma instituição hospitalar de ensino por meio da realização do exame físico pelo enfermeiro.

\section{MÉTODOS}

Trata-se de um estudo transversal, realizado com amostra por conveniência de 202 idosos hospitalizados numa clínica médica, em um hospital de ensino de médio porte de um município do estado do Paraná (Brasil), no período de setembro de 2017 a janeiro de 2018.

Os critérios utilizados para seleção da amostra foram:

a. Ter idade acima ou igual a 60 anos;

b. Estar hospitalizado por no mínimo 24 horas na instituição no período da coleta de dados;

c. Possuir capacidade cognitiva para participar do estudo9.

No caso de idosos sem condições cognitivas para responder às questões da pesquisa, na etapa da entrevista o cuidador/familiar foi convidado a participar; para este foram elencados os seguintes critérios de inclusão:

a. Ter idade igual ou superior a 18 anos;

b. Ser cuidador/familiar;

c. Residir com o idoso há, pelo menos, três meses.

Inicialmente, a coleta de dados realizada na unidade de internação contemplou o rastreio cognitivo por meio do Mini Exame do Estado Mental (MEEM). O instrumento possui 11 itens agrupados em sete categorias, representadas por grupos de funções cognitivas específicas: orientação temporal, orientação espacial, memória imediata, atenção e cálculo, memória de evocação, linguagem e capacidade construtiva visual ${ }^{10}$. A pontuação varia de zero a 30 , sendo os seguintes pontos de corte para avaliação: 13 pontos para analfabetos; 18 pontos, escolaridade baixa e média; e 26 pontos, escolaridade alta9.

$\mathrm{Na}$ sequência, com o objetivo de classificar e caracterizar a amostra, foi elaborado questionário sociodemográfico e clínico construído especificamente para o estudo. Os idosos foram submetidos ao exame físico de enfermagem que compreendeu: avaliação de pele e anexos dos diversos segmentos, couro cabeludo e cabelos, pele da face, turgor, temperatura da pele, espessura, textura, presença de cicatriz, sensibilidade da pele, prurido, unhas, medidas de nutrição (índice de massa corporal [IMC]) e lesões elementares primárias e secundárias. $\mathrm{O}$ instrumento foi construído com base em referências que abordam a semiologia da pele, com adaptação ao paciente idoso ${ }^{11-13}$.

A coleta de dados foi realizada por enfermeiras residentes em saúde do idoso e acadêmicas de enfermagem, bolsistas de iniciação científica, que foram capacitadas por uma enfermeira especialista em estomaterapia, com experiência clínica na área, por meio de 45 horas de atualização teórico-prática sobre avaliação de pele no idoso, a qual contemplou as seguintes temáticas: lesões elementares, lesão por pressão, lesão por fricção, lesão por adesivo, dermatites associadas à incontinência, prevenção e tratamento para lesão por pressão.

Os dados foram tabulados e analisados por meio do software Stata ${ }^{\circledR}$, versão 12. Estes foram submetidos à análise exploratória e descritos por meio de frequência absoluta e percentual, médias e seus desvios-padrão.

O projeto foi aprovado pelo Comitê de Ética da Universidade Estadual de Ponta Grossa com parecer $n^{\circ}$ 1.461.471 e mediante CAAE no 66782217.9.0000.5689. Foram respeitados os preceitos éticos de participação voluntária e consentida de cada sujeito, conforme a resolução vigente na época da realização da pesquisa.

\section{RESULTADOS}

Houve predomínio de sexo feminino $(\mathrm{n}=103 ; 51 \%)$ e faixa etária entre 60-69 anos $(\mathrm{n}=86 ; 42,5 \%)$, com média de idade de 71 anos $( \pm 0,61)$ (mínima de 60 e máxima de 98 anos) (Tabela 1). Dos participantes, 89 (44,2\%) estavam internados na clínica cirúrgica, 44 (21,8\%) tinham diagnóstico de doenças do aparelho circulatório (Classificação Internacional de Doenças [CID 10]) e $166(82,2 \%)$ ficaram internados entre 1-10 dias, com tempo médio de 7,1 dias $( \pm 0,59)$. Na avaliação do screening cognitivo pelo MEEM, constatou-se que $75(37,1 \%)$ participantes obtiveram score entre 19-25 pontos. As demais condições clínicas estão apresentadas na Tabela 1. 
Tabela 1. Distribuição das características sociodemográficas e clínicas de idosos internados em um hospital de ensino. Paraná, Brasil, 2018.

\begin{tabular}{|c|c|c|}
\hline & Variáveis & n (\%) \\
\hline \multirow{2}{*}{ Sexo } & Feminino & $103(51)$ \\
\hline & Masculino & 99 (49) \\
\hline \multirow{3}{*}{ Faixa etária } & $60-69$ anos & $86(42,5)$ \\
\hline & 70-79 anos & $76(37,7)$ \\
\hline & $>80$ anos & $40(19,8)$ \\
\hline \multirow{3}{*}{ Estado civil } & Casado & $105(52)$ \\
\hline & Solteiro & $24(11,9)$ \\
\hline & Viúvo & $73(36,1)$ \\
\hline \multirow{4}{*}{ Escolaridade* } & Alta & $20(10)$ \\
\hline & Média & $30(14,8)$ \\
\hline & Baixa & $120(59,4)$ \\
\hline & Analfabeto & $32(15,8)$ \\
\hline \multirow{3}{*}{ Cor da pele } & Branco & $147(72,8)$ \\
\hline & Pardo & $36(17,8)$ \\
\hline & Negro & $19(9,4)$ \\
\hline \multirow{4}{*}{ Setor de internação } & Clínica cirúrgica & $89(44,1)$ \\
\hline & Clínica médica & $76(37,6)$ \\
\hline & Clínica de neurologia & $33(16,3)$ \\
\hline & Clínica de infectologia & $4(2)$ \\
\hline \multirow{5}{*}{ Diagnóstico conforme CID 10** } & Doenças do aparelho circulatório & $44(21,8)$ \\
\hline & Doenças do aparelho respiratório & $32(15,9)$ \\
\hline & Doenças do aparelho digestivo & $26(12,9)$ \\
\hline & Doenças do sistema osteomuscular e do tecido conjuntivo & $28(13,9)$ \\
\hline & Outros & $72(35,5)$ \\
\hline \multirow{3}{*}{ Tempo de internação } & $1-10$ dias & $166(82,2)$ \\
\hline & $11-20$ dias & $23(11,4)$ \\
\hline & 21 dias ou mais & $13(6,4)$ \\
\hline \multirow{2}{*}{ Doença crônica } & $\operatorname{Sim}$ & $170(84,2)$ \\
\hline & Não & $32(15,8)$ \\
\hline \multirow{2}{*}{ Tabagismo } & $\mathrm{Sim}$ & $58(28,7)$ \\
\hline & Não & $144(71,3)$ \\
\hline \multirow{2}{*}{ Etilismo } & Sim & $32(15,8)$ \\
\hline & Não & $170(84,2)$ \\
\hline \multirow{2}{*}{ Dieta } & Oral & $174(86,1)$ \\
\hline & Enteral & $28(13,9)$ \\
\hline \multirow{2}{*}{ Uso de medicação } & $\mathrm{Sim}$ & $197(97,5)$ \\
\hline & Não & $5(2,5)$ \\
\hline \multirow{2}{*}{ Uso de dispositivos médicos } & $\mathrm{Sim}$ & $150(74,3)$ \\
\hline & Não & $52(25,7)$ \\
\hline \multirow{4}{*}{ Mobilidade } & Independente & $76(37,6)$ \\
\hline & Auxílio profissional & $35(17,3)$ \\
\hline & Uso de tecnologias*** & $20(10)$ \\
\hline & Restrito ao leito & $71(35)$ \\
\hline \multirow{4}{*}{ MEEM } & $0-13$ pontos & $58(28,7)$ \\
\hline & 14-18 pontos & $24(12)$ \\
\hline & 19-25 pontos & $75(37,1)$ \\
\hline & 26-30 pontos & $45(22,2)$ \\
\hline \multirow{5}{*}{ Escala de Braden } & $<9$ pontos & $07(3,5)$ \\
\hline & 10-12 pontos & $19(9,4)$ \\
\hline & 13-14 pontos & $30(14,8)$ \\
\hline & 15-18 pontos & $56(27,7)$ \\
\hline & 19-23 pontos & $90(44,5)$ \\
\hline
\end{tabular}

*Escolaridade: alta ( $\geq 8$ anos de estudo); média (4-8 anos incompletos); baixa (1-4 anos incompletos). **Diagnóstico "Outros": inclui os capítulos I, II, III, IV, V, VI, VII, XI, XIV, XX e XXI do CID 10. ***Uso de tecnologias: inclui andador, bengala e muleta. CID 10 = Classificação Internacional de Doenças; MEEM = Mini Exame do Estado Mental. Fonte: Os autores (2018). 
Quanto ao exame físico do sistema tegumentar, $128(63,4 \%)$ entrevistados apresentavam couro cabeludo/ cabelos limpos; 116 (57,4\%), pele com coloração normal; 160 (79,2\%), espessura fina; 101 (50\%), textura macia; 144 (71,3\%), cicatriz; $182(90,1 \%)$, sensibilidade; e 152 $(75,3 \%)$, prurido. A maioria $(\mathrm{n}=78 ; 38,6 \%)$ foi avaliada com IMC eutrófico (22-27). Constatou-se que 176 (87,1\%) participantes apresentavam alguma lesão elementar (Tabela 2).

Foram identificadas 364 lesões elementares primárias, das quais $236(64,8 \%)$ eram alterações de cor; 74 (20,4\%), coleções líquidas; e 54 (14,8\%), coleções sólidas. As lesões elementares secundárias totalizaram 324 , das quais

Tabela 2. Distribuição das características do exame físico tegumentar de idosos internados em um hospital de ensino. Paraná, Brasil, 2018.

\begin{tabular}{|c|c|c|}
\hline \multicolumn{2}{|c|}{ Variáveis } & \multirow{2}{*}{$\begin{array}{c}\text { n (\%) } \\
11(5,4)\end{array}$} \\
\hline \multirow{7}{*}{ Couro cabeludo/cabelos } & Caspa & \\
\hline & Seborreia & $4(2)$ \\
\hline & Alopecia & $4(2)$ \\
\hline & Oleoso & $13(6,4)$ \\
\hline & Seco & $29(14,4)$ \\
\hline & Sujo & $13(6,4)$ \\
\hline & Limpo & $128(63,4)$ \\
\hline \multirow{5}{*}{ Coloração } & Cianose & $3(1,5)$ \\
\hline & Rubor & $27(13,4)$ \\
\hline & Ictérica & $8(4)$ \\
\hline & Pálida & $48(23,8)$ \\
\hline & Normal & $116(57,4)$ \\
\hline \multirow{2}{*}{ Turgor } & Diminuído & $98(48,5)$ \\
\hline & Normal & $104(51,5)$ \\
\hline \multirow{2}{*}{ Temperatura } & Fria & $64(31,7)$ \\
\hline & Quente & $138(68,3)$ \\
\hline \multirow{2}{*}{ Espessura } & Fina & $160(79,2)$ \\
\hline & Grossa & $42(20,8)$ \\
\hline \multirow{2}{*}{ Textura } & Macia & $101(50)$ \\
\hline & Seca & $101(50)$ \\
\hline \multirow{2}{*}{ Presença de cicatriz } & $\mathrm{Sim}$ & $144(71,3)$ \\
\hline & Não & $58(28,7)$ \\
\hline \multirow{2}{*}{ Sensibilidade } & $\operatorname{sim}$ & $182(90,1)$ \\
\hline & Não & $20(9,9)$ \\
\hline \multirow{2}{*}{ Prurido } & $\operatorname{sim}$ & $152(75,3)$ \\
\hline & Não & $50(24,7)$ \\
\hline \multirow{7}{*}{ Unhas } & Transparentes & $28(14)$ \\
\hline & Lisas & $19(9,4)$ \\
\hline & Transparentes e lisas & $54(26,7)$ \\
\hline & Convexas & $15(7,4)$ \\
\hline & Côncavas & $15(7,4)$ \\
\hline & Manchas & $32(15,8)$ \\
\hline & Baqueteamento & $39(19,3)$ \\
\hline \multirow{3}{*}{$\mathrm{IMC}$} & $<22$ & $56(27,7)$ \\
\hline & $22-27$ & $78(38,6)$ \\
\hline & $>27$ & $68(33,7)$ \\
\hline \multirow{2}{*}{ Apresenta lesão } & Sim & $176(87,1)$ \\
\hline & Não & $26(12,9)$ \\
\hline Apresenta lesões elementares & $\operatorname{Sim}$ & $152(75,3)$ \\
\hline Primárias & Não & $50(24,7)$ \\
\hline Apresenta lesões elementares & $\operatorname{Sim}$ & $151(74,7)$ \\
\hline Secundárias & Não & $51(25,3)$ \\
\hline
\end{tabular}

*Índice de massa corporal (IMC): < 22 baixo peso; $22-27$ eutrófico; > 27 sobrepeso. Fonte: Os autores (2018). 
158 (48,8\%) eram alterações de textura e espessura e 166 $(51,2 \%)$, perdas teciduais. Lesões elementares são alterações no tegumento cutâneo determinadas por processos inflamatórios, degenerativos, circulatórios ou neoplásicos, por distúrbios do metabolismo ou por defeitos de formação ${ }^{11}$. As lesões elementares primárias são alterações de cor, coleções líquidas e coleções sólidas, já as lesões elementares secundárias são alterações de textura, espessura e perdas teciduais ${ }^{11}$.

\section{DISCUSSÃO}

Entre os participantes, verificou-se predomínio do sexo feminino, em consonância com os resultados de pesquisas nacionais acerca de lesões em idosos hospitalizados ${ }^{5,14}$ e na comunidade $^{4}$. Alterações hormonais provenientes do processo de envelhecimento podem contribuir para o aparecimento das lesões de pele nas idosas ${ }^{8}$.

Para a variável idade, tanto a média como a faixa etária identificada são semelhantes à pesquisa descritiva e exploratória realizada com 55 idosos paraibanos hospitalizados, na qual foi constatada maior prevalência de lesões em idosos jovens $^{8}$; tal fato pode ser justificado pela procura tardia aos serviços de saúde ou falta de acompanhamento do serviço aos idosos mais velhos.

A análise do estado civil apontou maior proporção de idosos casados, como esperado para a faixa etária da população do estudo e identificado em uma investigação exploratória acerca da avaliação de pele em idosos hospitalizados, em que $50 \%$ dos participantes eram casados ${ }^{14}$. Compreende-se a condição casado como um aspecto positivo para o idoso no que diz respeito ao auxílio nas atividades de vida e instrumentais de vida diária, colaborando para ações de prevenção e cuidado da pele.

O expressivo percentual de idosos com baixa escolaridade identificado nesse estudo é semelhante aos resultados da pesquisa transversal, retrospectiva e por meio de dados registrados em 1.168 prontuários do Serviço do Arquivo Médico do Hospital de Clínicas da Universidade Federal do Triângulo Mineiro ${ }^{5}$, em que predominaram participantes com baixo nível de instrução. Uma possível explicação pode ser atribuída ao perfil da comunidade atendida pelo hospital, vinculado ao Sistema Único de Saúde, em que predominam idosos com condições econômicas desfavoráveis, que na sua grande maioria não tiveram acesso ao sistema educacional.
No que se refere à cor da pele branca, essa se torna mais suscetível a lesões tegumentares em razão de suas características histológicas, como menor proteção de melanina e menos fibras de colágeno ${ }^{15,16}$. Em consonância com os achados, destaca-se a pesquisa transversal realizada em Palmas com 259 idosos, dos quais 66,7\% possuíam cor da pele branca ${ }^{16}$.

Para as variáveis período e tempo médio de internação, os achados diferem de outros estudos nacionais, os quais identificaram maior tempo médio de internação $(12,7$ e 10 dias $)^{13,14}$, o que pode ser atribuído ao fato de os entrevistados estarem internados em setores considerados de alta rotatividade hospitalar.

A maioria dos idosos relatou doenças crônicas, uma condição esperada pela característica de a amostra ser com idosos hospitalizados. Autores apontam que a presença de lesões é mais prevalente entre os idosos com pelo menos uma doença crônica, com destaque para o diabetes, as doenças cardiovasculares e as imunossupressoras, as quais podem alterar o processo de cicatrização ${ }^{4}$. Os achados são semelhantes à pesquisa descritiva e exploratória integrante do Estudo Saúde, Bem-estar e Envelhecimento (SABE), desenvolvido para traçar as condições de vida e saúde dos idosos residentes no município de São Paulo, em que 54,7\% dos 2143 idosos possuíam doenças crônicas ${ }^{4}$.

Apesar de a maioria dos entrevistados ter relatado não ser tabagista ou etilista, esses hábitos são considerados problemas de saúde pública, pela alta mortalidade das doenças provenientes dessas condições ${ }^{17}$. Na pele, tais substâncias podem provocar a quebra dos tecidos, lesionando as células, resultando no envelhecimento precoce e interferindo na integridade da pele ${ }^{17}$.

Entre os participantes, constatou-se o predomínio de dieta por via oral. A nutrição é um dos fatores intrínsecos que afeta a pele e sua funcionalidade ${ }^{18}$. Autores indicam a associação entre nutrição e o retardo da cicatrização com o desenvolvimento de lesões ${ }^{19}$.

O elevado uso de medicação identificado nos idosos deste estud ${ }^{18}$ está em consonância com a pesquisa de coorte prospectiva realizada em um hospital de ensino com 215 idosos, a qual revelou que 69,7\% faziam uso de medicação ${ }^{20}$. Os fármacos podem provocar alterações que podem diminuir a sensibilidade da pele e provocar $\operatorname{alergias}^{20}$.

O uso de dispositivos médicos e de mobilidade pode contribuir para a ocorrência de traumas e restrições. Durante 
a internação hospitalar, o idoso pode estar sujeito ao uso de dispositivos necessários ao seu tratamento, como cateteres, tubos, drenos, sondas e outros. Contudo, os dispositivos médicos que possuem ação de aderência à pele favorecem o rompimento da integridade cutânea a partir da sua manipulação e remoção,já os de mobilidade causam impacto nos cuidados diários e na troca de posições ${ }^{3}$.

A alta pontuação obtida no screening cognitivo (MEEM) pelos idosos participantes pode ser atribuída à característica etária da amostra, composta predominantemente por idosos jovens e ativos. Contudo não foram encontradas investigações acerca do rastreio cognitivo em idosos com lesões de pele para comparação com os achados.

$\mathrm{Na}$ realização do exame físico tegumentar, observou-se a escassez de estudos acerca da temática, especificamente no segmento etário idoso. Contudo, compreende-se a relevância da identificação e do rastreio precoce de lesões de pele por meio do exame físico tegumentar pela equipe de enfermagem.

No contexto hospitalar, os idosos apresentaram elevado número de lesões cutâneas, com destaque para as denominadas elementares, definidas como qualquer modificação estrutural da pele e que possuem diferentes causas, tais como: circulatória, hiperplásica, metabólica, inflamatória e degenerativa ${ }^{21}$.

Dentre as lesões elementares, constatou-se o predomínio de alterações de cor, as quais afetam a epiderme e a derme, não possuem relevo ou consistência e ocorrem por vasodilatação, constrição ou extravasamento de hemácias e por diminuição ou aumento da melanina ou depósitos de outros pigmentos e substâncias ${ }^{11}$. Do mesmo modo, houve elevada quantidade de alterações de textura e espessura e perda teciduais que podem ser oriundas da eliminação ou destruição patológica e de reparação em tecidos subcutâneos ${ }^{11}$.

O desenho transversal foi um fator limitante da avaliação entre as relações de causa e efeito. Além disso, a amostragem é representativa de uma comunidade local, de modo que não permite generalizar os resultados para outros territórios. Sugere-se que investigações realizadas com este segmento etário possam incluir a visita domiciliar para a coleta das informações, além de possíveis avaliações de conhecimentos dos profissionais sobre o assunto e medidas de atuação desses.

Aponta-se que a escassez de estudos epidemiológicos acerca de lesões na pele no segmento etário idoso dificultou a comparação dos achados da presente investigação, o que justifica a necessidade de realização de estudos epidemiológicos especialmente com essa população.

\section{CONCLUSÃO}

O estudo identificou a elevada prevalência de lesões de pele em idosos internados em uma instituição hospitalar de ensino por meio da realização do exame físico pelo enfermeiro. As lesões tegumentares podem interferir no estado funcional e contribuir para o aumento do tempo de internação e a mortalidade prematura.

Nesse contexto, considera-se importante que o profissional enfermeiro realize o exame físico da pele com avaliação criteriosa para identificação das alterações, sejam elas naturais ou patológicas, que o idoso possa apresentar, possibilitando, dessa forma, a construção de planos de cuidados gerontológicos voltados às necessidades específicas desse segmento etário.

\section{CONTRIBUIÇÃO DOS AUTORES}

Conceitualização, Grden CRB; Ivastcheschen T; Cabral LAP; Reche PM; Metodologia, Grden CRB; Ivastcheschen T; Reche PM; Oliveira de DAS; Investigação, Ivastcheschen T; Oliveira de DAS; Cabral LAP; Redação, Grden CRB; Ivastcheschen T; Bordin D; Redação - Revisão \& Edição, Grden CRB; Ivastcheschen T; Cabral LAP; Bordin D; Reche PM; Aquisição de Financiamento, Não se aplica; Supervisão, Grden CRB. 


\section{REFERÊNCIAS}

1. Universidade Federal do Maranhão - UNA-SUS/UFMA; Motta LB, organizadora. Conceitos básicos sobre envelhecimento. UNASUS/UFMA. São Luís, 2013.

2. Montanari T. Histologia: texto, atlas e roteiro de aulas práticas. 3a ed. Ponto Alegre: Edição do Autor; 2016.

3. Busanello J, Pinto DM, Schons ES, Baumgart D, Poll MA. Cuidados de enfermagem ao paciente adulto: prevenção de lesões cutaneomucosas e segurança do paciente. Rev Enferm da UFSM. 2015;5(4):597-606. https://doi. org/10.5902/2179769216310

4. Duim E, De Sá FHC, Duarte YAO, De Oliveira RCB, Lebrão $\mathrm{ML}$. Prevalence and characteristics of lesions in elderly people living in the community. Rev Esc Enferm USP. 2015;49(Speciallssue):50-6. https://doi.org/10.1590/50080$\underline{623420150000700008}$

5. Chavaglia SRR, Ohl RIB, Ferreira LA, Santiago VR, Abdanur AF, Soares AS. Caracterização de pacientes com lesão cutânea em unidades de internação médica e cirúrgica. Rev Enferm UFPE on line. 2015;9(1):183-92.

6. Mittag BF, Krause TCC, Roehrs H, Meier MJ, Danski MTR. Cuidados com lesão de pele: ações da enfermagem. ESTIMA, Braz J Enterestomal Ther. 2017;15(1):19-25. https:// doi.org/10.5327/Z1806-3144201700010004

7. Queiroz DB, Oliveira LC, Araújo CM, Reis LA. Perfil de internações de idosos em uma clínica de neurociências de um hospital público. Rev Enferm Contemp. 2016;5(1):16-24. https://doi.org/10.17267/2317-3378rec.v5i1.441

8. Bezerra AF, Silva JB, Silva JSN, Vigolvino LP, Meneses LBA. Lesões de pele em idosos hospitalizados: estudo de ocorrência. Anais do IV Congresso Internacional de Envelhecimento Humano; 2015 Set 21-26; Campina Grande. 2015;2(1).

9. Bertolucci PH, Brucki SM, Campacci SR, Juliano Y. The MiniMental State Examination in an outpatient population: influence of literacy. Arq Neuro-psiquiatr. 1994;52(1):1-7. https://doi.org/10.1590/S0004-282X1994000100001

10. Folstein MF, Folstein SE, McHugh PR. "Mini-mental state": a practical method for grading the cognitive state of patients for the clinician. J Psychiatr Res. 1975;12(3):189-98. https:// doi.org/10.1016/0022-3956(75)90026-6

11. Bolognia JL, Jorizzo JL, Rapini RP. Dermatologia. 2a ed. Rio de Janeiro: Elsevier; 2011.

12. Giaretta VMA, Silva AM, Renó ACM, Aguiar DAF, Arantes CMS, Posso MBS. Proposta de uma escala para avaliar o turgor da pele de idosos. Rev Cienc Saude. 2016;1(1):1-7.

13. Silva CFR, Santana RF, Oliveira BGRB, Carmo TG. High prevalence of skin and wound care of hospitalized elderly in Brazil: a prospective observational study. BMC Research Notes. 2017;10(81). https://doi.org/10.1186/s13104-0172410-6

14. Chibante CLP, Santo FHE, Santos TD. Perfil de clientes hospitalizados com lesões cutâneas. Rev Cuba Enferm. 2015;31(4).

15. Kohl E, Steinbauer J, Landthaler M, Szeimies RM. Skin ageing. J Eur Acad Dermatol Venereol. 2011;25(8):873-84. https:// doi.org/10.1111/j.1468-3083.2010.03963.x

16. Silva LMT. Idosos hospitalizados em risco de desenvolver lesão por pressão: contribuição do enfermeiro [trabalho de conclusão de curso]. Niterói: Universidade Federal Fluminense, Curso de Enfermagem; 2016.

17. Carvalho A, Borda CC, Moreira DM, Pereira MAR, Mário RF, Zychar BC. Envelhecimento cutâneo induzido pelo tabagismo. Atas de Ciências da Saúde. 2016;3(3).

18. Murphree RW. Impairments in skin integrity. Nurs Clin North Am. 2017;52(3):405-17. https://doi.org/10.1016/j. cnur.2017.04.008

19. Oliveira KDL, Haack A, Fortes RC. Terapia nutricional na lesão por pressão: revisão sistemática. Rev Bras Geriatr Gerontol. 2017;20(4). https://doi.org/10.1590/198122562017020.160195

20. Vieira CPB, Sá MS, Madeira MZA, Luz MHBA. Caracterização e fatores de risco para úlceras por pressão na pessoa idosa hospitalizada. Rev Rene. 2014;15(4):650-8. https://doi. org/10.15253/2175-6783.2014000400012

21. Porto CC. Semiologia médica.7a ed. Rio de Janeiro: Guanabara-Koogan; 2014. 